\title{
Exercise hormone irisin mitigates endothelial barrier dysfunction and microvascular leakage-related diseases
}

\author{
Jianbin Bi, ${ }^{1,2}$ Jia Zhang, ${ }^{1,2}$ Yifan Ren, ${ }^{1,2}$ Zhaoqing Du, ${ }^{1,2}$ Yuanyuan Zhang, ${ }^{3}$ Chang Liu, ${ }^{2}$ Yawen Wang, ${ }^{4,5}$ \\ Lin Zhang, ${ }^{5}$ Zhihong Shi, ${ }^{6}$ Zheng $\mathbf{W u},{ }^{2}$ Yi Lv, ${ }^{1,2}$ and Rongqian $\mathbf{W u}^{1}$ \\ ${ }^{1}$ National Local Joint Engineering Research Center for Precision Surgery \& Regenerative Medicine, Shaanxi Provincial Center \\ for Regenerative Medicine and Surgical Engineering, ${ }^{2}$ Department of Hepatobiliary Surgery, ${ }^{3}$ Department of Pediatrics, \\ ${ }^{4}$ Biobank, ${ }^{5}$ Department of Laboratory Medicine, and ${ }^{6}$ Department of Respiratory Medicine, The First Affiliated Hospital of \\ Xi'an Jiaotong University, Xi'an, Shaanxi Province, China.
}

Increased microvascular leakage is a cardinal feature of many critical diseases. Regular exercise is associated with improved endothelial function and reduced risk of cardiovascular disease. Irisin, secreted during exercise, contributes to many health benefits of exercise. However, the effects of irisin on endothelial function and microvascular leakage remain unknown. In this study, we found that irisin remarkably strengthened endothelial junctions and barrier function via binding to integrin $\alpha \mathrm{V} \beta 5$ receptor in LPS-treated endothelial cells. The beneficial effect of irisin was associated with suppression of the Src-MLCK- $\beta$-catenin pathway, activation of the AMPK-Cdc42/Rac1 pathway, and improvement of mitochondrial function. In preclinical models of microvascular leakage, exogenous irisin improved pulmonary function, decreased lung edema and injury, suppressed inflammation, and increased survival. In ARDS patients, serum irisin levels were decreased and inversely correlated with disease severity and mortality. In conclusion, irisin enhances endothelial barrier function and mitigates microvascular leakage-related diseases.

Conflict of interest: The authors have declared that no conflict of interest exists

Copyright: () 2020, American Society for Clinical Investigation.

Submitted: January 9, 2020

Accepted: June 3, 2020

Published: June 9, 2020.

Reference information: /CI Insight. 2020;5(13):e136277.

https://doi.org/10.1172/jci.

insight.136277.

\section{Introduction}

Microvascular leakage is a pivotal pathological process in many diseases, such as asthma, sepsis, acute respiratory distress syndrome (ARDS), anaphylaxis, and diabetic retinopathy. Increased endothelial permeability is the main cause of microvascular leakage (1). On one hand, endotoxin, inflammatory factors, and neutrophils facilitate endothelial myosin light chain (MLC) phosphorylation to combine with actin and induce the phosphorylation of $\beta$-catenin and the separation of VE-cadherin from the cytoskeleton, resulting in disruption of adhesion junctions between endothelial cells $(2,3)$. On the other hand, Rho GTPase family members dynamically regulate intercellular junctions and cytoskeletal remodeling via the formation of cortical actin. Under disease conditions, decreased activation of Cdc42 and Rac1 and increased activation of Rho lead to cytoskeletal remodeling, resulting in endothelial cell shrinkage, intercellular broadening, and ultimately increased vascular permeability $(4,5)$. Additionally, mitochondrial dysfunction, including decreased mitochondria quantity, imbalanced mitochondrial dynamics, mitochondrial fragmentation, respiratory chain inhibition, and massive ROS generation, directly damages endothelial cells and increases pulmonary endothelial permeability (6-8).

It has been widely reported that regular exercise can improve endothelial function and slow the progression of atherosclerosis (9). Irisin, a newly identified hormone secreted by skeletal muscle during exercise, was initially discovered as a myokine responsible for browning white fat (10). Subsequent studies have shown that irisin is implicated in type 2 diabetes, obesity, aging, and mitochondrial function (11). A recent study verified that integrin $\alpha \mathrm{V} \beta 5$ is the receptor of irisin in osteocytes and fat cells (12). Vascular integrins are major mediators of endothelial adhesion to extracellular matrix (13). Src, downstream pathway of vascular integrins, directly alters the structure of the endothelial barrier by phosphorylation of MLCK, $\beta$-catenin, and focal adhesion (14). Several studies have reported that the effects of irisin in improved energy metabolism are associated with activation of adenosine monophosphate activated protein kinase (AMPK), the central metabolic sensor $(15,16)$. Interestingly, in addition to regulating energy metabolism, AMPK 
activation can also strengthen the aggregation of microtubules and myosin to protect vascular barrier function (17-19). Previous studies have shown that mitochondrial ATP generation regulates endothelial cytoskeletal remodeling by Rac activation (20). However, the role of irisin in microvascular endothelial permeability remained unknown. We therefore hypothesized that irisin strengthened endothelial junctions and barrier function via binding to integrin $\alpha \mathrm{V} \beta 5$ receptor, further inhibiting the phosphorylated Src $(\mathrm{P}-\mathrm{Src})$ (Y416)/P-MLCK (Y464)/P- $\beta$-catenin (Y142) pathway and activating the AMPK-mitochondria-Cdc42/ Rac1 pathway in endothelial cells. The main purpose of this study is to explore whether irisin benefits the endothelial barrier function. Additionally, this study also sought to clarify the therapeutic effect of irisin on microvascular leakage-related diseases.

\section{Results}

Irisin strengthened endothelial junctions and barrier function. Phalloidin and VE-cadherin were stained to assess cytoskeletal remodeling and adherens junction integrity in endothelial cells, respectively. These results showed that LPS induced massive formation of actin stress fibers and intercellular gaps due to cell contractions in human microvascular endothelial cells (HMVECs). These changes were largely reduced by irisin treatment (Figures 1, A and B). Meanwhile, Transwell permeability assays were performed to verify endothelial cell permeability. Irisin treatment significantly decreased the LPS-induced increase in FITC-labeled albumin in HMVECs (Figure 1C). Additionally, endothelial cell permeability was assessed by transendothelial electrical resistance (TER) in endothelial cell monolayers. TER measurement and determination of the maximum TER relative to baseline revealed that $10 \mathrm{nM}$ irisin significantly increased the TER in HMVECs (Figure 1D). Moreover, irisin treatment largely reversed the decreasing trend in TER after LPS challenge in HMVECs (Figure 1E). Similar results were found in HUVECs. Irisin decreased the formation of actin stress fibers and enhanced the VE-cadherin- and $\beta$-catenin-mediated adherens junction (Figure $1 F)$. Furthermore, the irisin-treated HUVECs showed an increasing trend in TER and decreasing endothelial cell permeability (Figure 1, G-J).

Exogenous irisin administration alleviated microvascular leakage-related diseases. At 24 hours after LPS administration intratracheally and 21 hours after CLP operation, serum irisin levels were decreased in LPSand CLP-treated mice compared with control mice, while exogenous irisin administration significantly increased serum irisin levels $(P<0.05$, Figure 2A). TEM analysis showed distinct increases in joint gaps between pulmonary microvascular endothelial cells, whereas irisin treatment abolished this change to a great extent (Supplemental Figure 1; supplemental material available online with this article; https://doi. org/10.1172/jci.insight.136277DS1). Levels of total cells and total proteins in the bronchoalveolar lavage fluid (BALF) increased greatly after LPS administration intratracheally, while exogenous irisin treatment immediately or 6 hours after LPS administration intratracheally showed significant reductions (Figure 2, $\mathrm{B}$ and $\mathrm{C})$. H\&E staining of the lung tissues showed a mass of alveolar hemorrhage, inflammatory cell infiltration and alveolar wall thickening after LPS administration. Irisin treatment immediately or 6 hours after LPS administration significantly alleviated these changes. Consistent with these histological changes, irisin treatment significantly decreased the acute lung injury (ALI) scores and water content compared with the control-treated animals in the above models (Figure 2, D-F). Arterial blood gas analysis showed that irisin treatment immediately or 6 hours after LPS administration reversed the decrease in partial pressure of oxygen $\left(\mathrm{PaO}_{2}\right)$ and increase in $\mathrm{PaCO}_{2}$ levels at 24 hours after LPS administration intratracheally (Figure 2, $\mathrm{G}$ and $\mathrm{H}$ ). Meanhile, we found that irisin neutralizing antibody pretreatment further increased the levels of total cells and proteins in the BALF, aggravated tissue damage and decreased $\mathrm{PaO}_{2}$ after LPS administration (Figure 2, B-H). To further verify the protective effect of irisin, we used clp-, gut ischemia reperfusion- (IR-), and aged rat liver IR-induced ALI models. The results showed that irisin significantly relieved liver tissue damage and reduced the levels of total cells, total proteins, and inflammatory cytokines after LPS administration, CLP, gut IR, and aged rat liver IR induction. Meanwhile, low concentration of irisin treatment $(50 \mu \mathrm{g} / \mathrm{kg})$ showed limited effects after CLP operation (Figure 2, I-M; Supplemental Figure 2; and Supplemental Figure 3, B-G). In addition, mice treated with $250 \mu \mathrm{g} / \mathrm{kg}$ exogenous irisin had a higher survival rate than saline-treated mice after CLP operation, although there was no difference in weight loss between untreated and treated mice (Figure $2 \mathrm{~N}$ and Supplemental Figure $3 \mathrm{~A}$ ).

Blood cell analysis revealed a significant increase in inflammatory cell number in BALF 24 hours after LPS treatment. Administration of irisin significantly reduced WBC, lymphocyte, monocyte, and neutrophil counts in the BALF (Supplemental Figure 4, A-D). MPO immunostaining also showed a lower percentage 
A
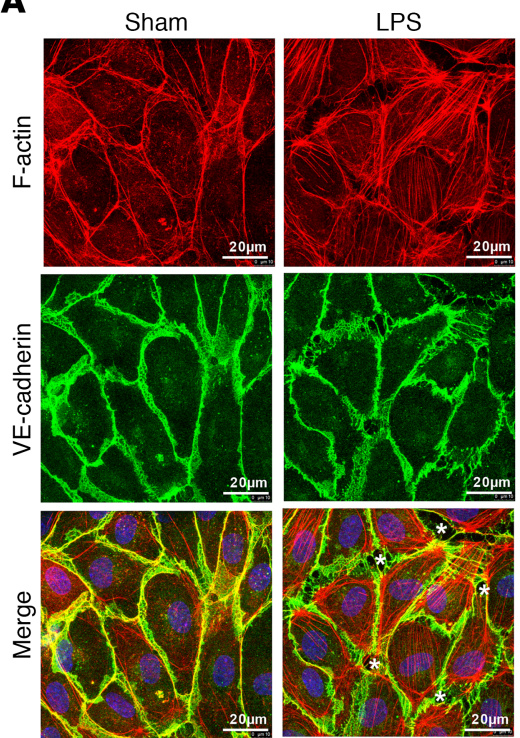

$\mathbf{F}$
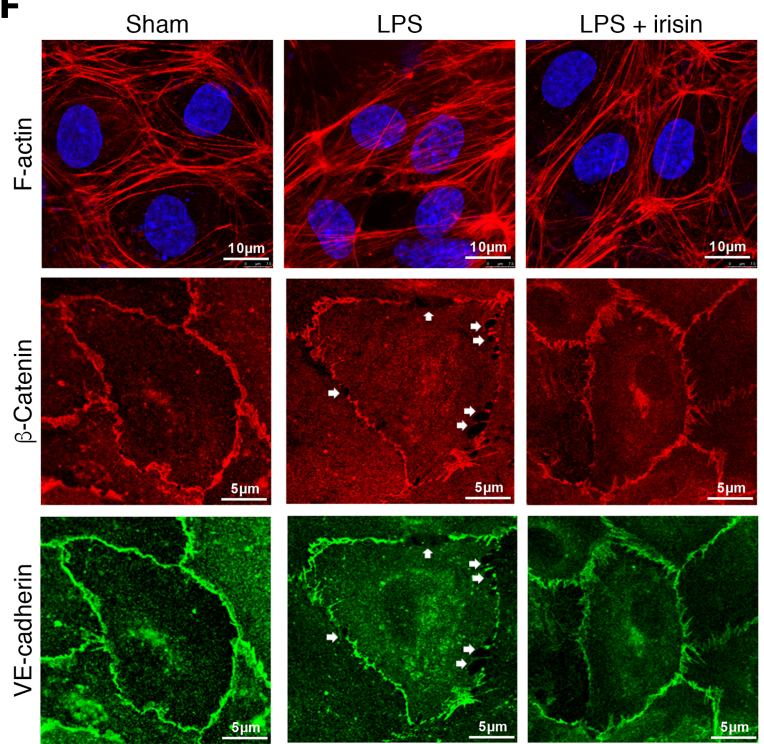

B
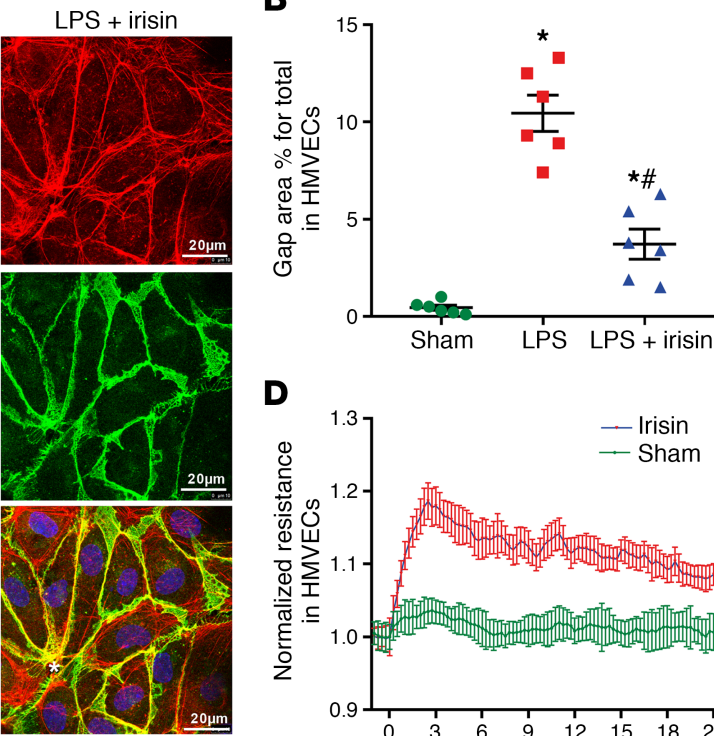

D

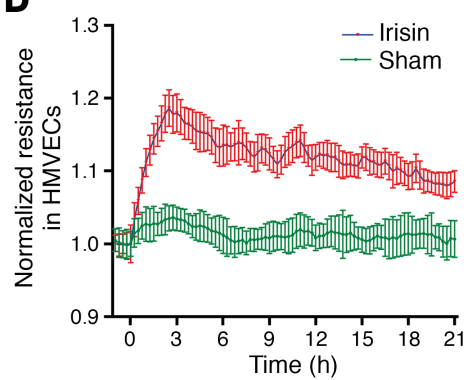

G

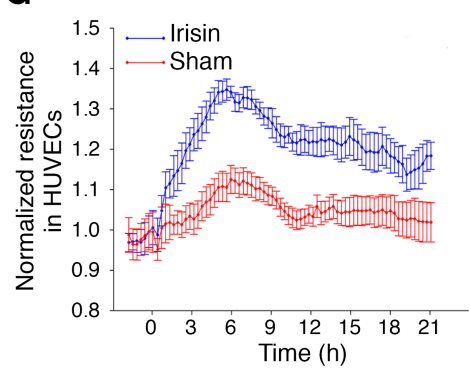

I

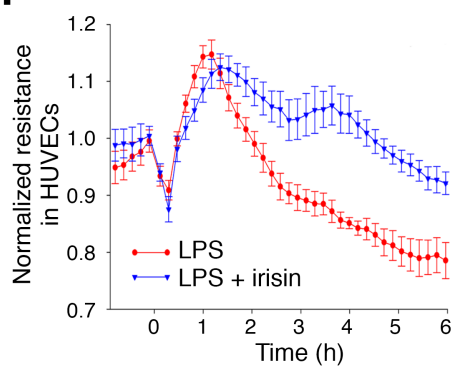

C

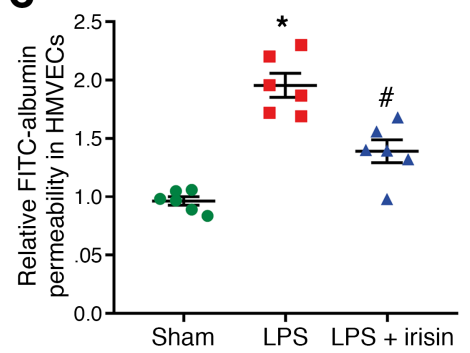

E

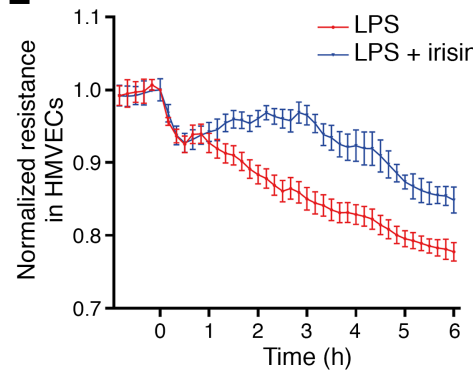

H

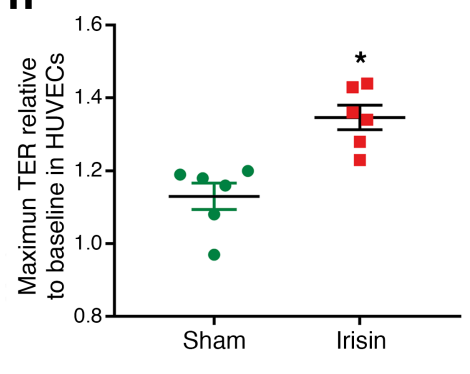

J

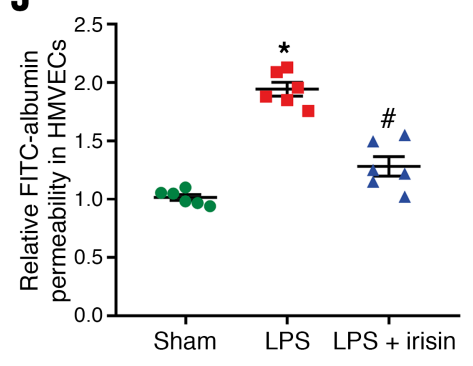

Figure 1. Irisin strengthened endothelial barrier function and reduced LPS-induced endothelial cell hyperpermeability. HMVECs and HUVECs were treated with $10 \mathrm{nM}$ irisin immediately after $500 \mathrm{ng} / \mathrm{mL}$ LPS administration. (A) Phalloidin and VE-cadherin staining for assessing cytoskeletal remodeling and adherens junction integrity in HMVECs 2 hours after LPS and irisin treatment. Scale bar: $20 \mu \mathrm{m}$. Asterisks in the Phalloidin and VE-cadherin staining represent gaps between the cells. (B) Gap areas in the total areas. (C) Relative permeability of FITC-labeled albumin 2 hours after LPS and irisin treatment in HMVECs. (D) Transendothelial electrical resistance (TER) in irisin- and control-treated human microvascular endothelial cells (HMVECs). (E) TER in irisinand control-treated HMVECs after $500 \mathrm{ng} / \mathrm{mL}$ LPS administration. (F) Phalloidin (scale bar: $10 \mu \mathrm{m}$ ), VE-cadherin (scale bar: $5 \mu \mathrm{m}$ ), and $\beta$-catenin (scale bar: $5 \mu \mathrm{m}$ ) staining for assessment of cytoskeletal remodeling and adherens junction integrity in HUVECs 2 hours after LPS and irisin treatment. Arrows in the VE-cadherin/ $\beta$-catenin staining represent gaps between the cells. (C) TER in irisin- and control-treated HUVECs. (H) Maximum TER relative to baseline. (I) TER in irisin- and control-treated HUVECs after $500 \mathrm{ng} / \mathrm{mL}$ LPS administration. (J) Relative permeability of FITC-labeled albumin 2 hours after LPS and irisin treatment in HUVECs. $n=6$ per group, mean \pm SEM, ${ }^{*} P<0.05$ versus the sham group, ${ }^{\#} P<0.05$ versus the LPS group. The $t$ test was used to analyze the differences between 2 groups $(\mathbf{H})$, and 1-way ANOVA was used to analyze the differences among 3 or more groups (B, C, and J).

of $\mathrm{MPO}^{+}$cells in the lungs of irisin-treated mice compared with vehicle-treated mice (Supplemental Figure 4, E and F). The levels of TNF- $\alpha$, MIP-2, and CIRP were determined in the BALF; irisin treatment markedly reduced the increases in the levels of these inflammatory factors after LPS administration (Supplemental Figure 4, G-I). The serum levels of TNF- $\alpha$, MIP-2, and CIRP showed similar results, and serum IL-10 levels largely increased after irisin treatment in LPS-treated mice (Supplemental Figure 4, J-M). 
A

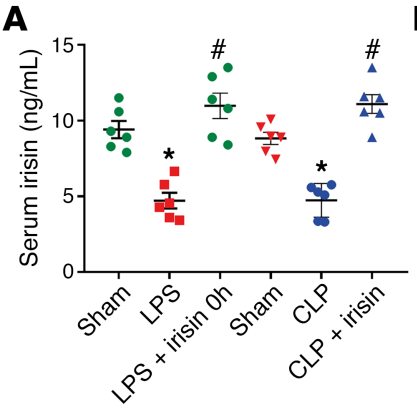

B C

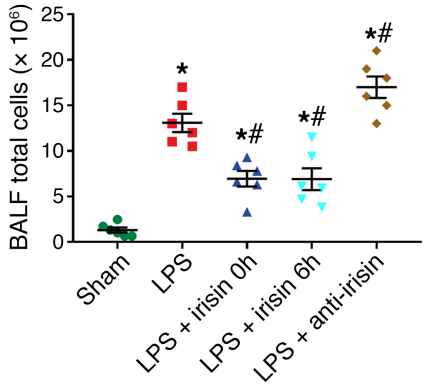

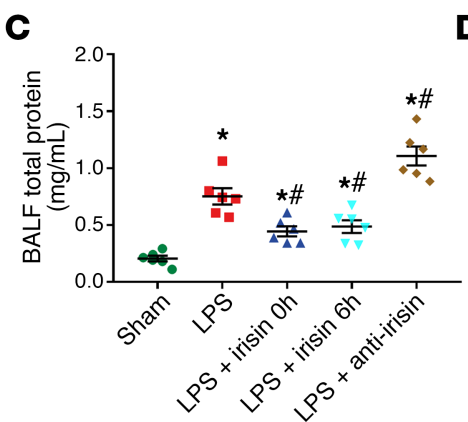

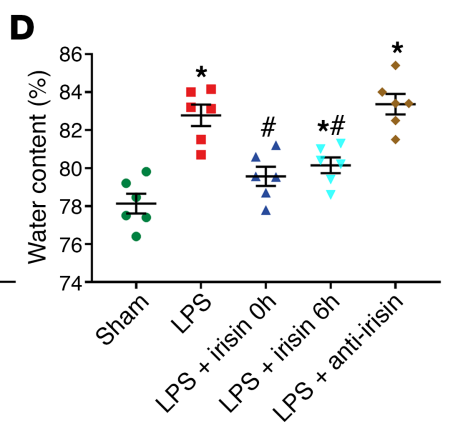

E

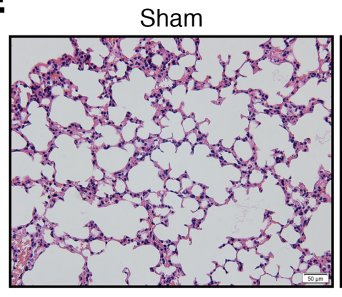

$\mathbf{F}$

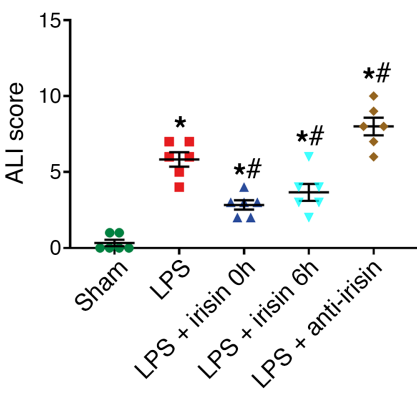

G

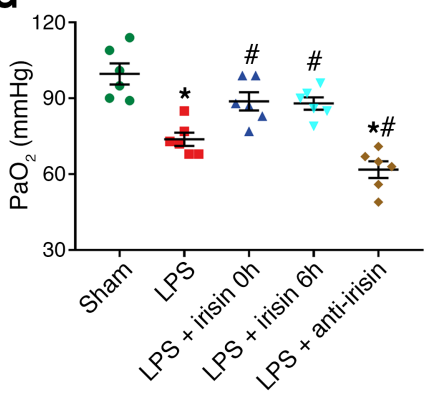

H
LPS + irisin Oh LPS + irisin $6 h$

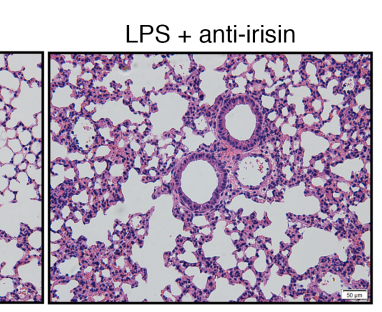

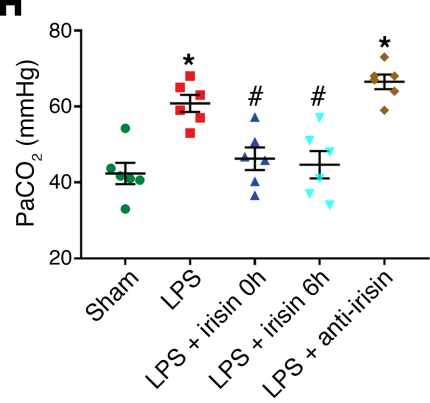

I

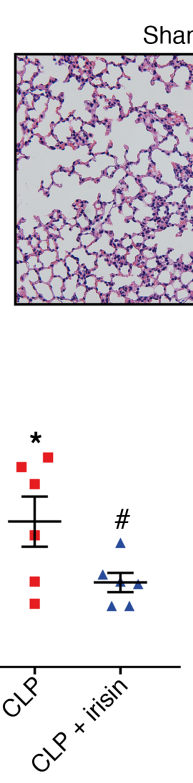

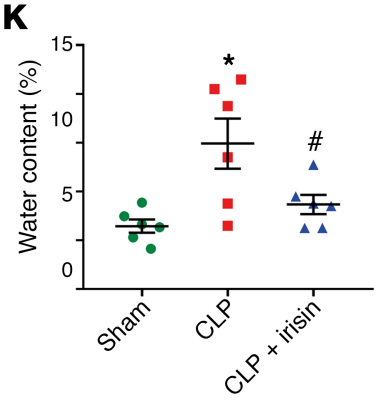

L

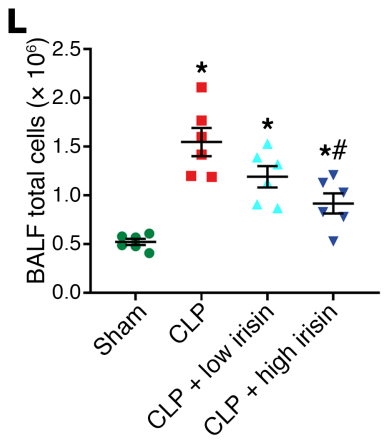

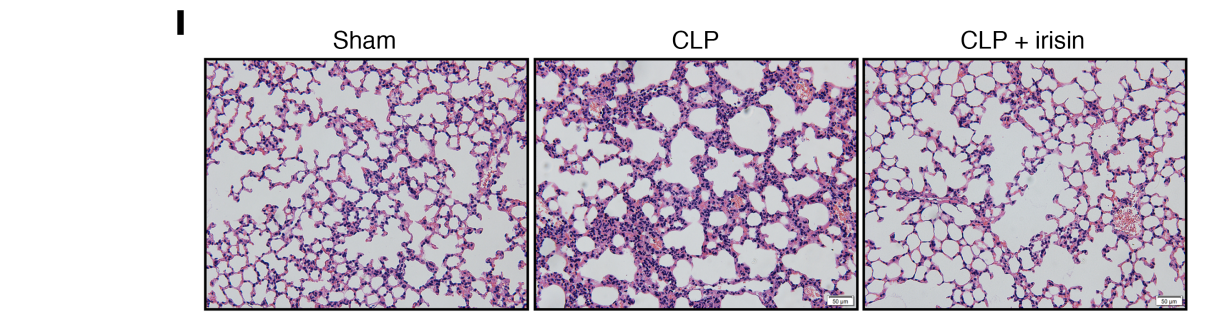

Figure 2. Exogenous irisin administration alleviated microvascular leakage-related diseases. Irisin was given by i.v. administration ( $250 \mu \mathrm{g} / \mathrm{kg}$, a single dose) immediately or 6 hours after LPS administration intratracheally $(2 \mathrm{mg} / \mathrm{kg})$, and immediately after CLP operation. Irisin neutralizing antibody was administrated by i.v. injection in mice ( $50 \mu \mathrm{g} / \mathrm{kg}$, a single dose) 24 hours before LPS was administrated intratracheally. Vehicle group of mice was given equivalent amounts of saline. At 24 hours after LPS was administrated intratracheally or 21 hours after CLP operation, lung tissue, BALF, and arterial blood samples were collected. (A) Serum irisin levels. (B and C) Total cells and protein levels in BALF in LPS-induced lung microvascular leakage. (D) Water content of lungs. (E) H\&E staining in LPS-induced lung microvascular leakage. Scale bar: $50 \mu \mathrm{m}$. (F) ALI scores. (C) At 24 hours after LPS administration, arterial blood was obtained from the abdominal aorta, and $\mathrm{PaO}_{2}$ was assessed via blood gas analyzer. (H) PaCO, levels. (I) H\&E staining of lung in CLP-induced sepsis. Scale bar: 50 $\mu \mathrm{m}$. (J) ALI score. (K) Water content. (L and $\mathbf{M}$ ) Total cells and protein levels in BALF in CLP-induced sepsis. (N) Seven-day survival study in CLP-induced sepsis. Kaplan-Meier curves were used for survival analysis and log-rank testing for difference analysis. High irisin represents a dose of $250 \mu \mathrm{g} / \mathrm{kg}$. Low irisin represents a dose of $50 \mu \mathrm{g} / \mathrm{kg} ; n=6$ per group, mean \pm SEM. ${ }^{*} P<0.05$ versus the sham group, ${ }^{\#} P<0.05$ versus the LPS or CLP group. One-way ANOVA was used to analyze the differences between groups. 
Irisin enhanced endothelial cell barrier function via binding to integrin $\alpha V \beta 5$ receptor and suppression of the P-Src (Y416)/P-MLCK (Y464)/P- $\beta$-catenin (Y142) pathway. Immunofluorescent staining showed an observable colocalization of irisin and integrin $\alpha \mathrm{v} \beta 5$ proteins in irisin-treated HMVECs (Figure 3A). Co-IP analysis demonstrated that irisin could directly bind to the integrin $\alpha \mathrm{V} \beta 5$ receptor in the lung (Figure 3B). Cilengitide trifluoroacetate, an inhibitor of integrin $\alpha \mathrm{V} \beta 5$, was used to further confirm whether irisin enhanced endothelial cell barrier via integrin $\alpha \mathrm{V} \beta 5$. We found that cilengitide trifluoroacetate alone induced obvious gap formation. Meanwhile, cilengitide trifluoroacetate abolished the protective function of irisin in decreasing albumin permeability and intercellular gap areas in HMVECs (Figure 3, C-E). Additionally, Consistent with the in vitro experiments, the cilengitide trifluoroacetate-treated mice lost therapeutic effects of irisin in reducing exudation of proteins and cells, as well as water content, in the lungs (Figure 3, F-H). Western blot showed that irisin significantly decreased the phosphorylation of Src, MLCK, and $\beta$-catenin at Tyr416, Tyr464, and Tyr142, respectively, after LPS treatment (Figure 3, I-L). Meanwhile, cilengitide trifluoroacetate abolished the protective role of irisin in decreasing P-Src, P-MLCK, and P- $\beta$-catenin (Figure 3, M-P).

Irisin restored endothelial barrier function by activation of AMPK-Rac1/Cdc42 Signaling. AMPK phosphorylation at Thr172 was downregulated after LPS challenge, whereas irisin treatment significantly increased the activation of AMPK in HMVECs. Additionally, despite the reduction of Rac1 and Cdc42 activation after LPS administration, irisin treatment markedly increased activation of both Rac1 and Cdc42 in HMVECs (Figure 4, A-D). Furthermore, cilengitide trifluoroacetate markedly reversed the increased activation of AMPK after irisin administration (Figure 4, E and F). To further determine the relationship between AMPK phosphorylation and activation of Rac1 and Cdc42, AMPK siRNA was transfected. The protective effects of irisin in upregulating the activation of Rac1 and Cdc42 were abolished in HMVECs transfected with AMPK siRNA at 2 and 8 hours after LPS treatment (Figure 4, G-I). Fluorescence staining showed that HMVECs transfected with AMPK siRNA markedly increased the formation of actin stress fibers and intercellular gaps compared with normal HMVECs after LPS and irisin treatment (Figure 4, J and K). Similarly, AMPK siRNA-treated HMVECs exhibited lower TER and higher FITC-labeled albumin permeability in contrast to nonspecific siRNA-treated HMVECs (Figure 4, L and M).

Compound $C$ abolished the protective effects of irisin on LPS-induced microvascular leakage. A prominent increase in AMPK phosphorylation at Thr172 was observed in irisin-treated mice after LPS treatment, which completely abolished the reduction in LPS-treated mice (Figure 5, A and B). Administration of compound C, an AMPK inhibitor, decreased the activation of Rac1 after irisin treatment in LPS-induced microvascular leakage (Figure 5, C and D). Meanwhile, total cell and protein levels in the BALF were dramatically increased after compound C treatment (Figure 5, E-G). Additionally, mice that received compound $\mathrm{C}$ showed more serious histological changes, higher water content, lower $\mathrm{PaO}_{2}$ levels, and higher $\mathrm{PaCO}_{2}$ levels compared with the control-treated mice (Figure 5, H-L). Moreover, ATP production was significantly increased after irisin treatment, while compound $\mathrm{C}$ reversed this change in LPS- induced microvascular leakage (Figure 5M).

Irisin protected mitochondrial function in endothelial cells. Mitochondrial dysfunction of vascular endothelial cells is a pivotal pathologic mechanism of endothelial cell hyperpermeability. The expression of mitochondrial biogenesis-related PPAR $\gamma$ coactivator $1 \alpha$ (PGC-1 $\alpha)$ and mitochondrial transcription factor (TFAM) were decreased after LPS challenge in HMVECs. Irisin treatment significantly upregulated PGC-1 $\alpha$ and TFAM expression (Figure 6, A-C). Furthermore, HMVECs transfected with AMPK siRNA showed marked reduction of PGC-1 $\alpha$ and TFAM expression at 2 and 8 hours after LPS and irisin treatment (Figure 6, D and E). To determine the overall number of mitochondria, MitoTracker staining of live HMVECs was performed. Irisin markedly restored the LPS-induced decrease in mitochondria number. However, AMPK siRNA treatment significantly abolished the protective function of irisin in mitochondrial biogenesis (Figure 6, F and G). Additionally, ATP biosynthesis-related protein ATP synthase $\beta$ (ATPB) and mitophagy-related PTEN-induced putative kinase 1 (PINK-1) expression levels were increased after irisin administration (Figures 6, H-J). Consistent with Western blot results, ATP levels were significantly increased in irisin-treated HMVECs 2 and 8 hours after LPS challenge (Figure 6K). Furthermore, significant increases in uncoupling protein 2 (UCP2) expression were found after irisin treatment (Figure 6, L and M). ROS generation (detected by dihydroethidium [DHE] staining) showed that irisin treatment markedly decreased ROS fluorescence intensity in LPS-treated HMVECs (Figure 6, N and $\mathrm{O})$. Meanwhile, irisin markedly inhibited the expression of mitochondrial fission-related proteins dynamin related protein 1 (drp-1) and fission 1 (Fis-1) in LPS-treated HMVECs (Supplemental Figures 5). 
A
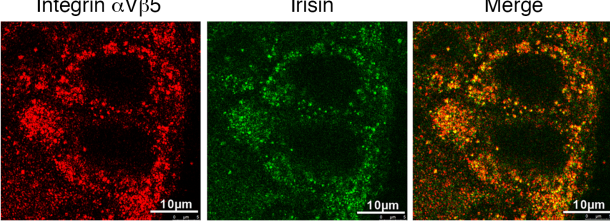

B

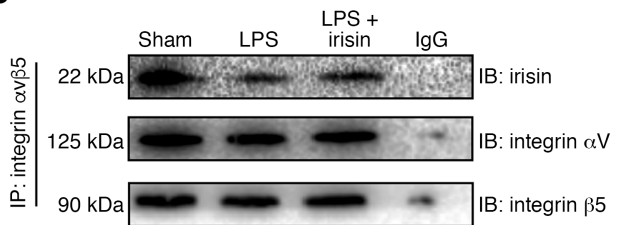

C

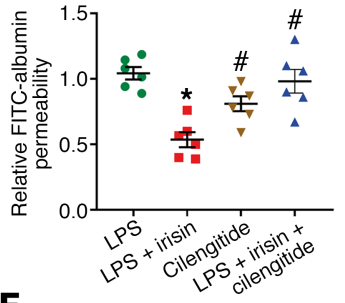

D

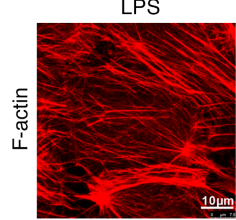

LPS + irisin
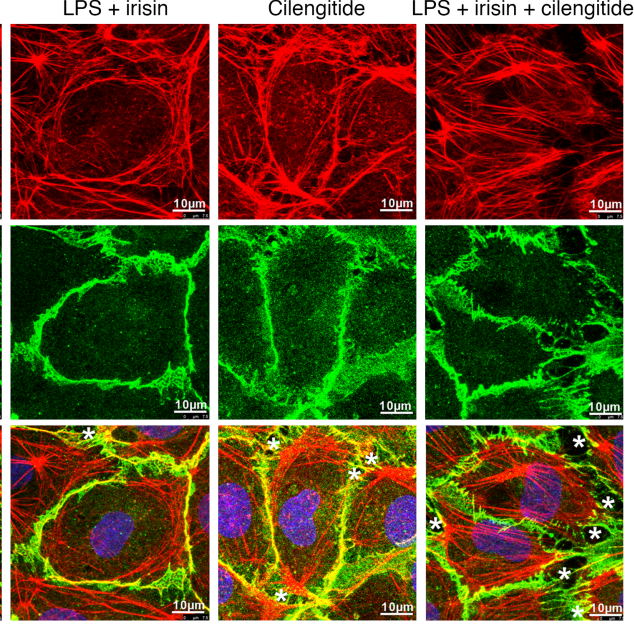

E

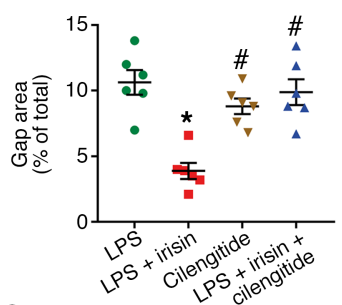

G

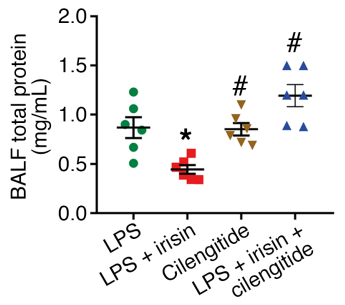

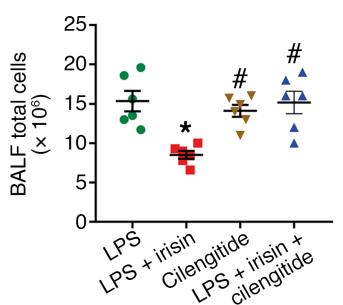

H

$\mathbf{K}$
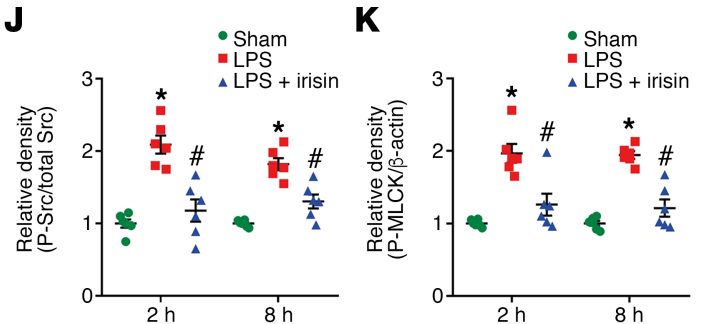

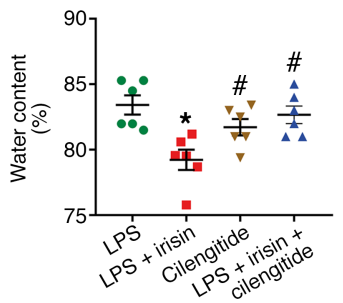

$\mathbf{L}$

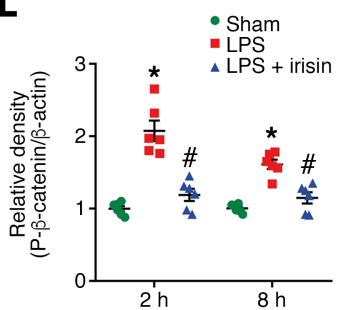

M
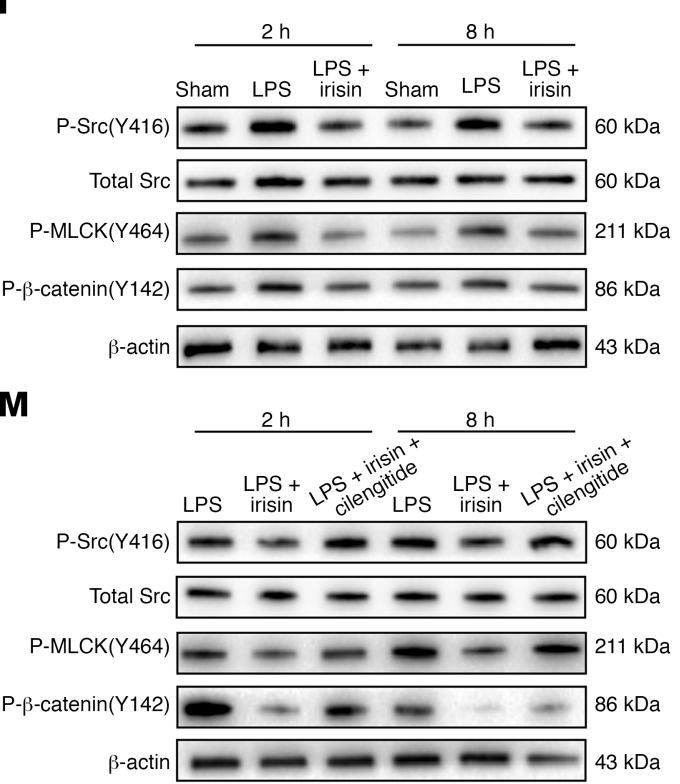

$\mathbf{N}$

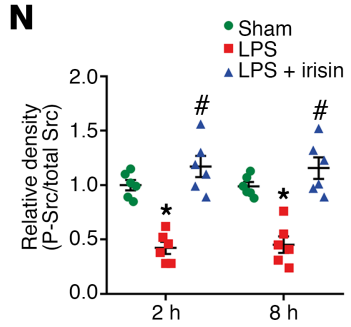

0

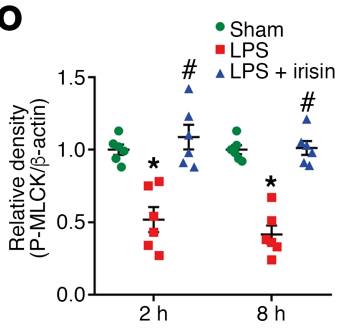

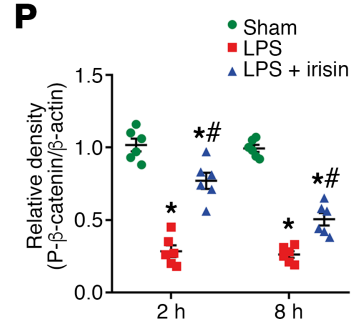

Figure 3. Irisin enhanced endothelial cell barrier function via binding to integrin $\alpha$ V $\beta 5$ receptor and suppression of P-Src (Y416)/P-MLCK (Y464)/P- $\beta$ catenin (Y142) pathway. Irisin ( $250 \mu \mathrm{g} / \mathrm{kg}$, i.v.) and cilengitide trifluoroacetate (20 mg $/ \mathrm{kg}$, i.v.) were given by i.v. administration immediately after LPS administration intratracheally $(2 \mathrm{mg} / \mathrm{kg}$ ). Vehicle group of mice was given equivalent amounts of saline. Lungs were harvested 24 hours after LPS administration. HMVECs were treated with $10 \mathrm{nM}$ irisin and $20 \mu \mathrm{M}$ cilengitide trifluoroacetate immediately after $500 \mathrm{ng} / \mathrm{mL}$ LPS administration. (A) Immunofluorescence colocalization of intergrin $\alpha \mathrm{V} \beta 5$ and irisin at 2 hours after LPS and irisin treatment in HMVECs. Scale bar: $10 \mu \mathrm{m}$. (B) Co-IP of irisin and integrin $\alpha \mathrm{V} \beta 5$ at 24 hours after LPS-induced microvascular leakage. (C) Relative permeability of FITC-labeled albumin 2 hours after LPS, irisin, and cilengitide trifluoroacetate treatment in HMVECs. (D) Phalloidin and VE-cadherin staining for assessing cytoskeletal remodeling and adherens junction integrity. Scale bar: $10 \mu \mathrm{m}$. Asterisks in the Phalloidin and VE-cadherin staining represents gaps between the cell. (E) Gap areas in the total areas. (F and $\mathbf{G})$ Total cells and protein levels in BALF in LPS-induced microvascular leakage. (H) Water content of lungs in LPS-induced microvascular leakage. (I-L) Western blot analysis of phosphorylation of Src, MLCK, and $\beta$-catenin at Tyr416, Tyr464, Tyr142, respectively. ${ }^{*} P<0.05$ versus the sham group, ${ }^{\#} P<0.05$ versus the LPS or CLP group. (M-P) Western blot analysis of phosphorylation of Src, MLCK, and $\beta$-catenin after treatment with cilengitide trifluoroacetate. ${ }^{*} P<0.05$ versus the LPS group, ${ }^{\#} P<0.05$ versus the irisin group; $n=6$ per group, mean \pm SEM. One-way ANOVA was used to analyze the differences between groups. 
A

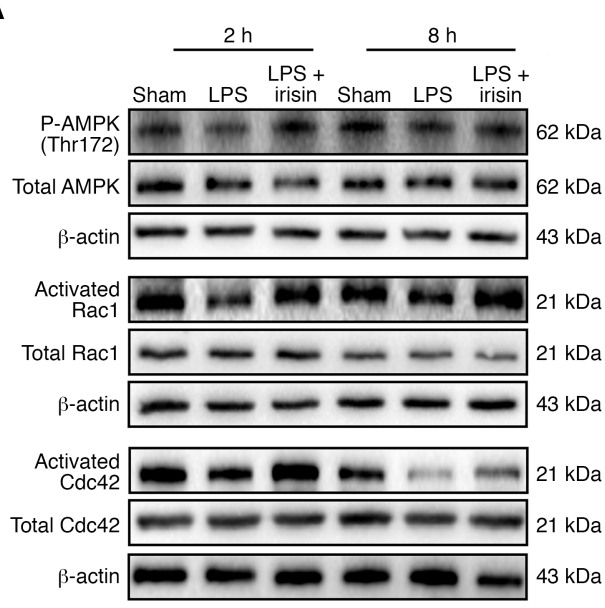

G

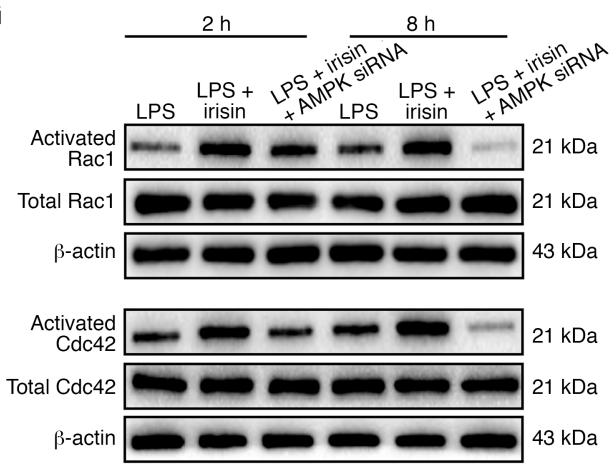

H

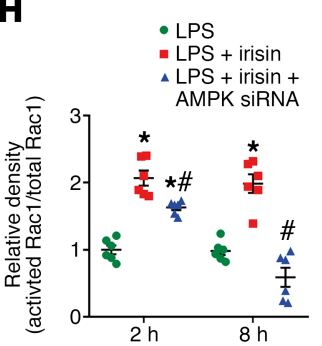

I

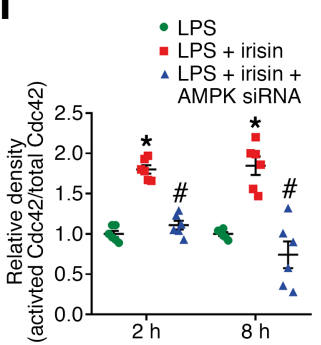

B

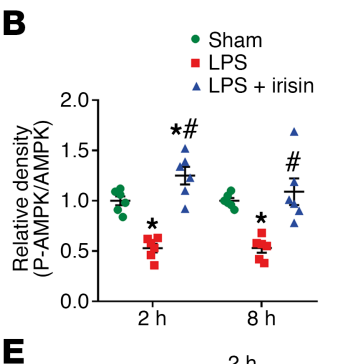

C
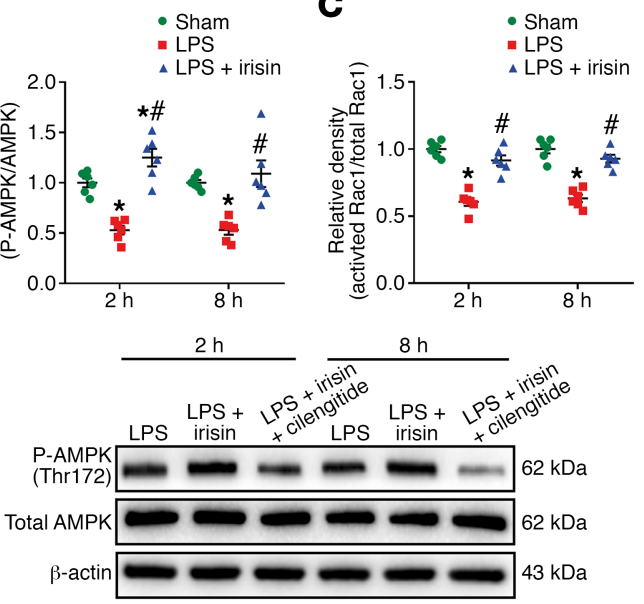

J
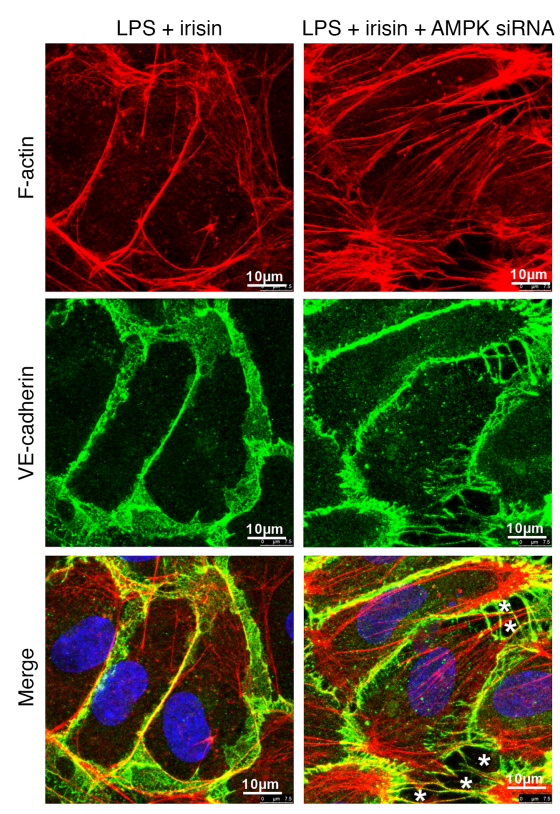

D

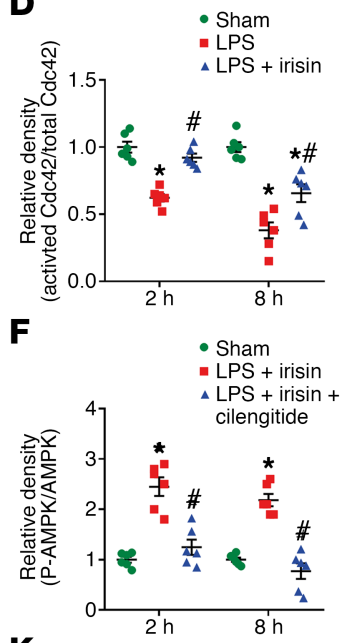

$\mathbf{K}$

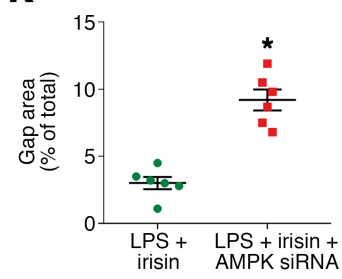

$\mathbf{L}$

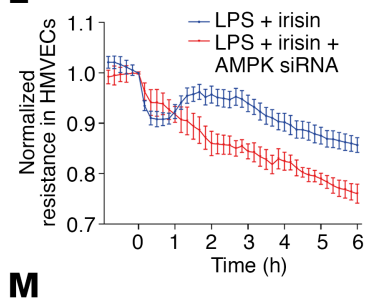

M

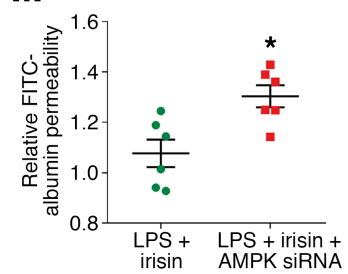

Figure 4. Irisin restored endothelial barrier function by activation of AMPK-Rac1/Cdc42 signaling. HMVECs were treated with $10 \mathrm{nM}$ irisin immediately after $500 \mathrm{ng} / \mathrm{mL}$ LPS administration. (A-D) Western blot analysis of activation of adenosine monophosphate-activated protein kinase (AMPK), as well as Rac1 and Cdc42 activation assays in irisin- and control-treated HMVECs. (E and F) Western blot analysis of activation of AMPK in cilengitide trifluoroacetate- and control-treated HMVECs after $500 \mathrm{ng} / \mathrm{mL}$ LPS and $10 \mathrm{nM}$ irisin administration. (G-I) Rac1 and Cdc42 activation assays in irisin- and LPS-treated HMVECs transfected with nonspecific siRNA or AMPK siRNA. (J) Phalloidin and VE-cadherin staining in HMVECs. Scale bar: $10 \mu \mathrm{m}$. Asterisks in the Phalloidin and VE-cadherin staining represents gaps between the cells. (K) Gap areas in the total areas. (L) TER. (M) Relative permeability of FITC-labeled albumin. $n=6$ per group, mean $\pm \mathrm{SEM},{ }^{*} P<0.05$ versus the sham group or the LPS + irisin group. ${ }^{\sharp} P<0.05$ versus the LPS or LPS + irisin group. The $t$ test was used to analyze the differences between 2 groups (K and $\mathbf{M})$, and 1-way ANOVA was used to analyze the differences among 3 or more groups (B-D, $\mathbf{F}, \mathbf{H}$, and $\mathbf{I})$.

Serum irisin levels were decreased and negatively correlated with disease severity and mortality in ARDS patients. Blood samples from 60 ARDS patients and 60 healthy volunteers were collected, patient demographics were documented, and serum irisin levels were measured (Supplemental Table 1). As shown in Figure 7A, serum irisin levels were decreased in ARDS patients compared with healthy volunteers $(P<0.05)$. Additionally, serum irisin levels were negatively correlated with Acute Physiology and Chronic Health Evaluation II (APACHE II) scores $\left(R^{2}=0.1336, P=0.004\right.$, Figure 7B). Meanwhile, there is a weak correlation between serum irisin and Sequential Organ Failure Assessment (SOFA) scores $\left(R^{2}=0.0687, P=0.045\right.$, Figure 7C). The survival analysis showed that patients with serum irisin levels $\geq 2.75 \mathrm{ng} / \mathrm{mL}$ had a lower mortality (Figure 7D). The univariate analysis showed hypertension, SOFA score at admission, APACHE 
A

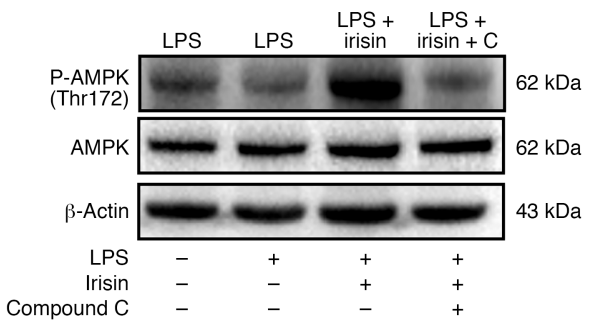

B

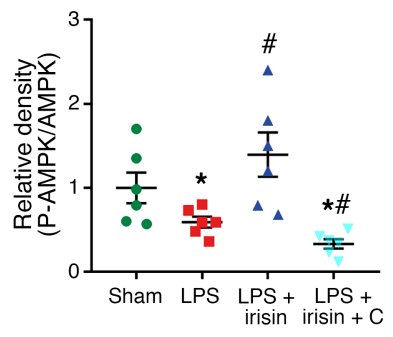

C

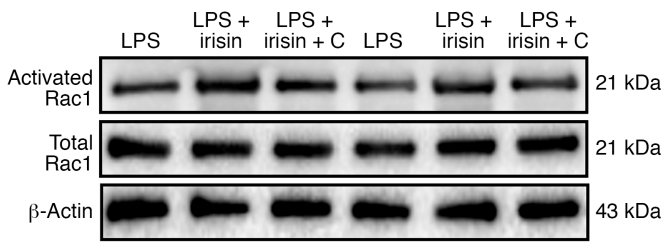

D

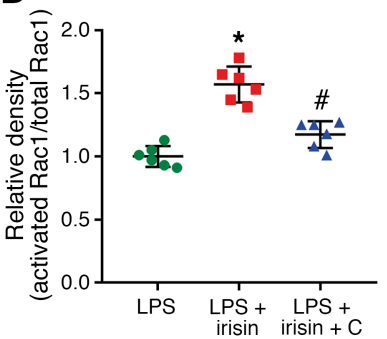

E

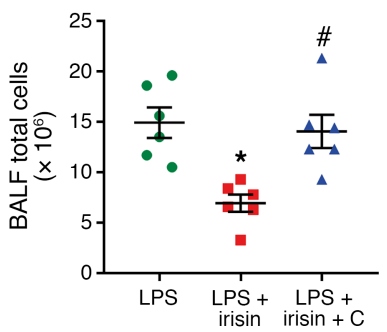

$\mathbf{F}$

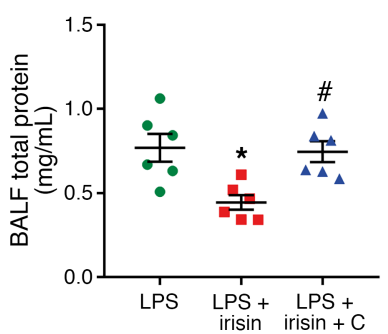

G

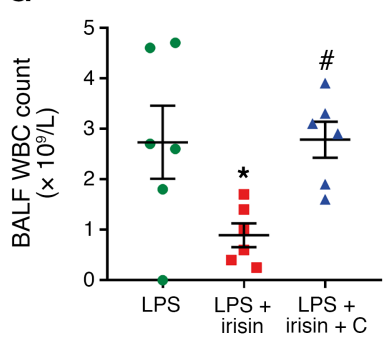

H

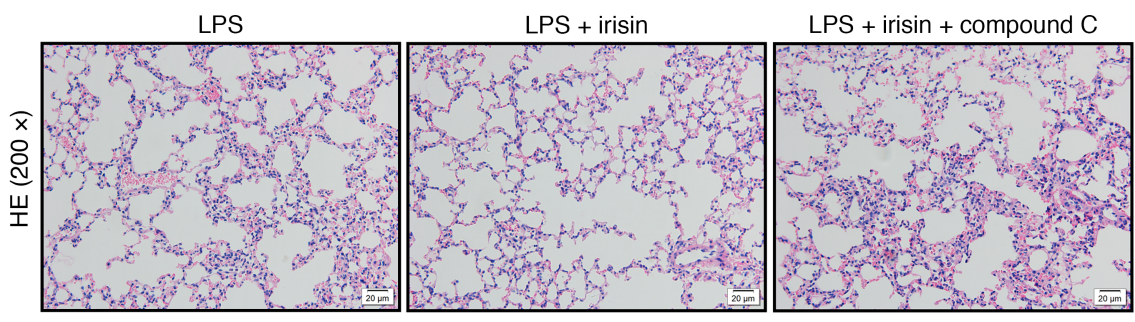

J

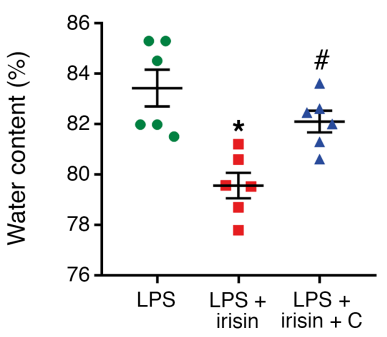

K

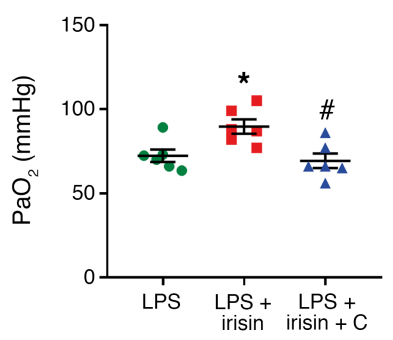

$\mathbf{L}$

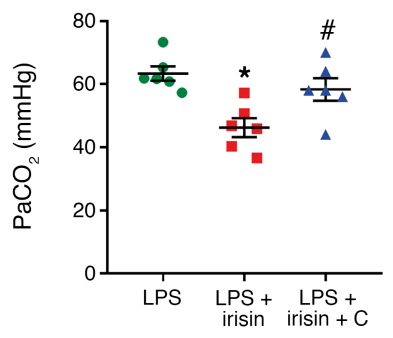

I

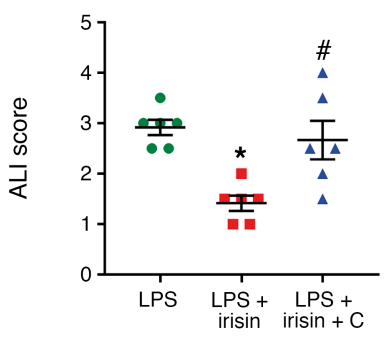

M

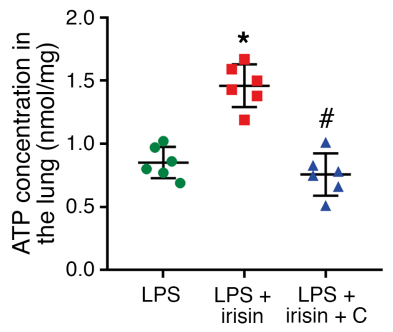

Figure 5. Compound C abolished the protective effects of irisin on LPS-induced microvascular leakage. Mice were treated with irisin (i.v., $250 \mu \mathrm{g} / \mathrm{kg}$, a single dose) and compound C ( $2 \mathrm{mg} / \mathrm{kg}$, an AMPK inhibitor) immediately after LPS administration. The vehicle group of mice was given equivalent amounts of saline. Twenty-four hours after LPS treatment, lung tissues, BALF, and arterial blood samples were collected. (A and B) Western blot analysis of the expression of AMPK and AMPK phosphorylation at Thr172. (C and D) Rac1 activation assays. (E-C) The total cells, total protein levels, and WBC numbers in BALF, respectively. (H) H\&E staining. Scale bar: $20 \mu \mathrm{m}$. (I) ALI score. (J) Water content in lungs. (K) PaO $2_{2}$ (L) PaCO ${ }_{2}$. (M) Lung ATP concentration in LPS-induced microvascular leakage. $n=6$ per group, mean $\pm \mathrm{SEM},{ }^{*} P<0.05$ versus the sham group, ${ }^{\#} P<0.05$ versus the LPS group. One-way ANOVA was used to analyze the differences between groups.

II score at admission, and serum irisin levels were associated significantly with 28-day mortality of ARDS patients. However, in the multivariate analysis, APACHE II score at admission (HR, 1.375; 95\% CI, 1.009$1.874 ; P=0.044$ ) and serum irisin levels (HR, $0.153 ; 95 \% \mathrm{CI}, 0.024-0.961 ; P=0.045]$ were independently associated with 28-day mortality of ARDS patients (Supplemental Table 2).

\section{Discussion}

In the present study, we found that low serum irisin was associated with worse outcomes in ARDS patients, and exogenous irisin protected against endothelial barrier dysfunction and microvascular leakage-related diseases via binding to integrin $\alpha \mathrm{V} \beta 5$ receptor, further inhibiting the P-Src (Y416)/P-MLCK 
A

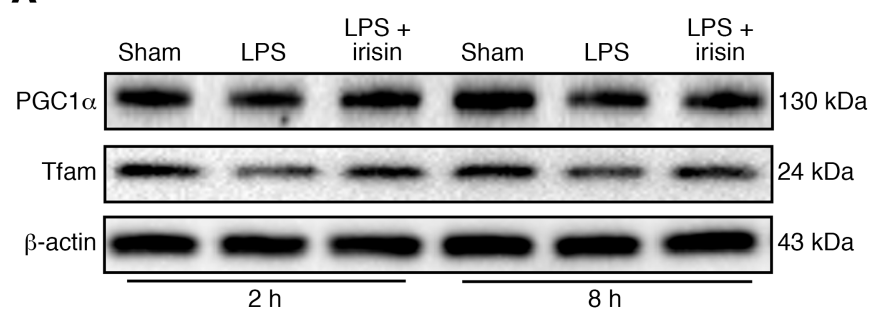

B

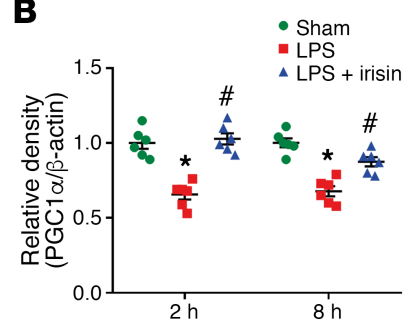

C

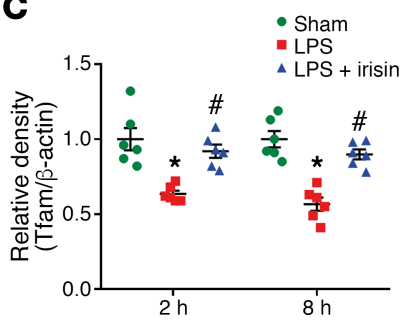

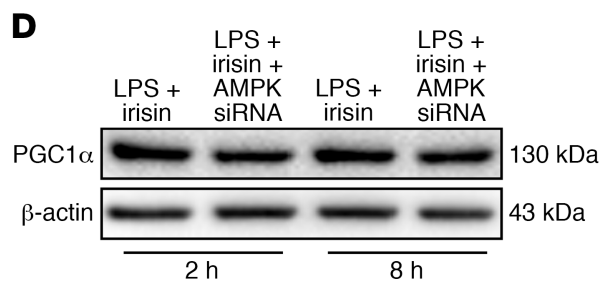

E

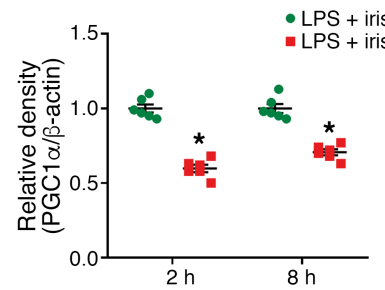

G
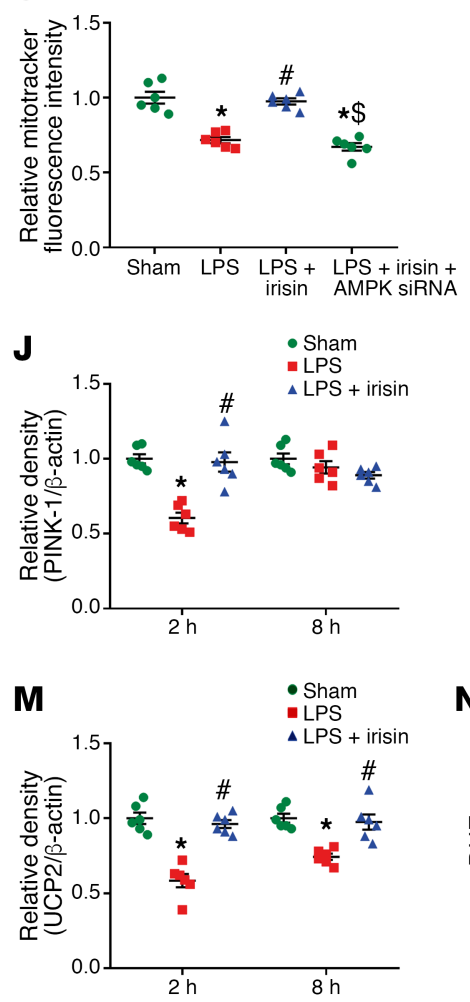

$\mathbf{F}$

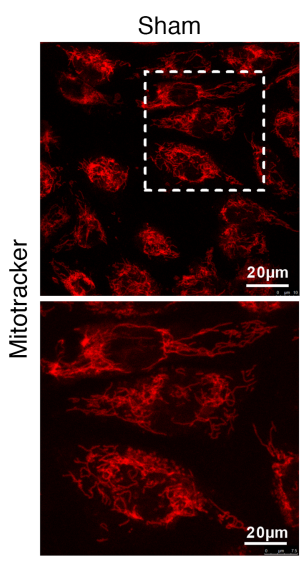

H

$$
\text { H }
$$

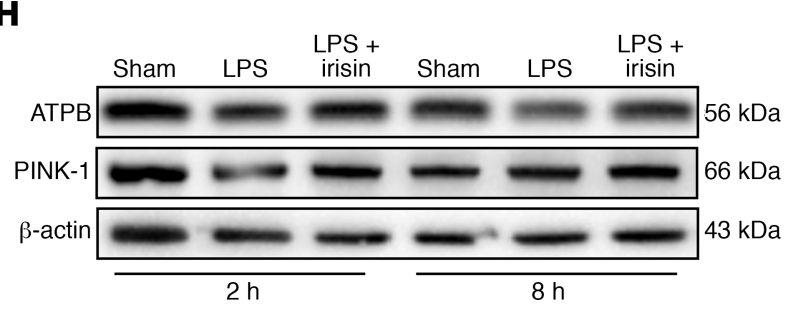

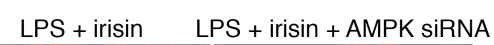
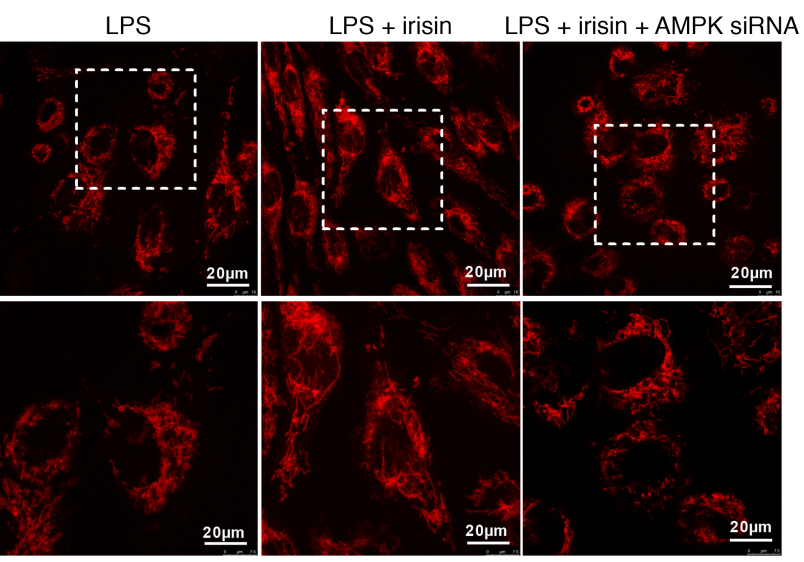
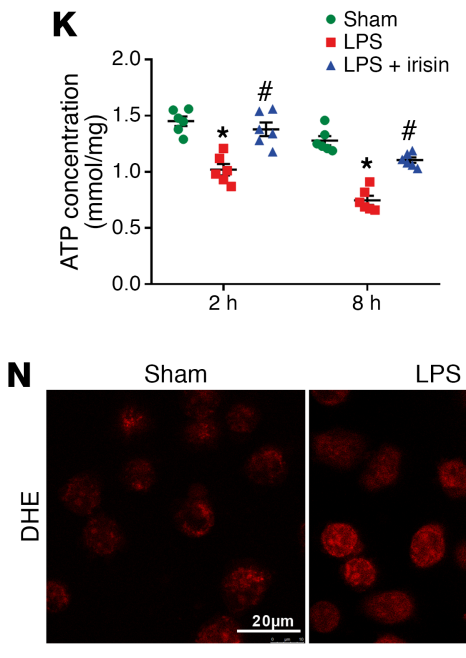

$\mathbf{L}$

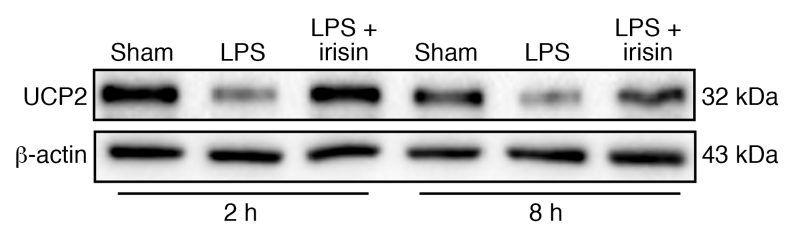

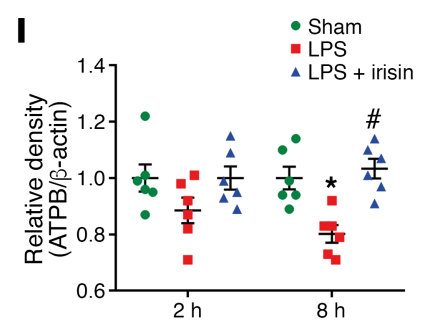

Figure 6. Irisin protected mitochondrial function in endothelial cells to restore endothelial barrier integrity. HMVECs were treated with 10 nM irisin immediately after $500 \mathrm{ng} / \mathrm{mL}$ LPS administration. (A-C) Western blot analysis of the expression of PPAR $\gamma$ coactivator $1 \alpha$ (PCC-1 $\alpha$ ) and mitochondrial transcription factor (TFAM) in HMVECs 2 hours and 8 hours after LPS treatment. (D and E) Western blot analysis of PGC-1 $\alpha$ expression in irisin- and LPS-treated HMVECs transfected with nonspecific siRNA or AMPK siRNA. (F and G) MitoTracker Red CMXRos fluorescence staining and fluorescence intensity of HMVECs 2 hours after LPS, irisin, and AMPK siRNA treatment. Scale bar: $10 \mu \mathrm{m}$. (H-J) Western blot analysis of the expression of ATP synthase $\beta$ (ATPB) and PTEN-induced putative kinase 1 (PINK1) in HMVECs. (K) ATP concentration 2 hours and 8 hours after irisin and LPS treatment in HMVECs. (L and $\mathbf{M}$ ) Western blot analysis of uncoupling protein 2 (UCP2) expression in HMVECs. ( $\mathbf{N}$ and $\mathbf{0}$ ) DHE fluorescence staining and its fluorescence intensity of HMVECs at 2 hours after LPS and irisin treatment. Scale bar: 20 m. $n=6$ per group, mean $\pm \mathrm{SEM},{ }^{*} P<0.05$ versus the sham group or LPS + irisin group, ${ }^{*} P<0.05$ versus the LPS group. One-way ANOVA was used to analyze the differences between groups. 
A
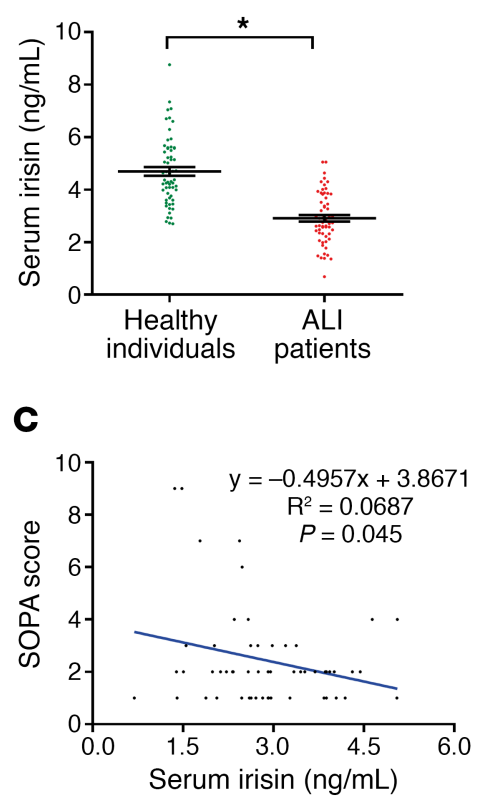

B

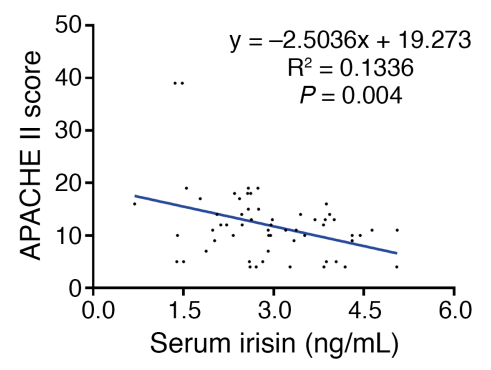

D

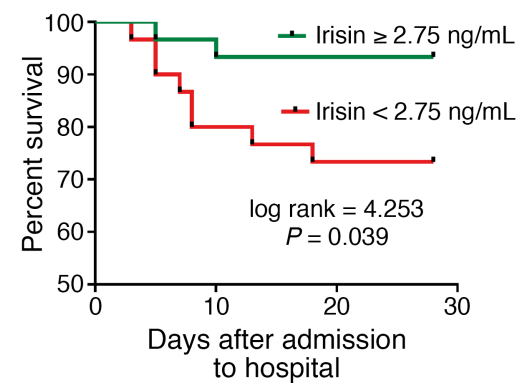

Figure 7. Serum irisin levels were decreased and negatively correlated with disease severity and mortality in ARDS patients. Blood samples from 60 ARDS patients and 60 healthy volunteers were collected, and serum irisin levels were measured. (A) Serum irisin levels in ARDS patients and healthy volunteers. The $t$ test was used to analyze the differences between 2 groups. (B) Correlation analysis of serum irisin and APACHE II scores. (C) Correlation analysis of serum irisin and SOFA scores; Spearman's correlation coefficient ( $\rho$ ) was used to analyze associations between 2 parameters. (D) Patients were divided into a high-irisin group and low-irisin group, based on the median of irisin concentration, and followed for 28 days after admission to the hospital to assess survival. Kaplan-Meier curves were used for survival analysis and log-rank testing for difference analysis. ${ }^{*} P<0.05$ compared with healthy volunteers.

(Y464)/P- $\beta$-catenin (Y142) pathway, activating the AMPK-Cdc42/Rac1 pathway, and improving mitochondrial function in endothelial cells (Figure 8). Irisin may, therefore, assist with the urgent medical need for preventing or minimizing ARDS and other microvascular leakage-related diseases.

Irisin, mainly secreted by the skeletal muscle during exercise, was initially discovered as a myokine responsible for the browning of white fat and thermogenesis in 2012 (10). Subsequent studies have shown that irisin regulates glucose/lipid metabolism and has antioxidant functionality in type 2 diabetes $(21,22)$. Additionally, irisin has shown protective effects on mitochondrial function in ischemia/reperfusion injury (23). Irisin, therefore, is anticipated to provide solutions for energy metabolism-related problems. A previous study compared lung injury before and after irisin administration in LPS-treated mice (24). In this study, we found that irisin remarkably strengthened endothelial junctions and barrier function via binding to integrin $\alpha \mathrm{V} \beta 5$ receptor in LPS-treated human endothelial cells. Serum irisin levels were decreased and negatively correlated with disease severity and mortality in ARDS patients, suggesting that irisin levels may predict the severity and prognosis of ARDS. More importantly, irisin showed dramatic therapeutic effects in multiple animal models of microvascular leakage-related diseases, suggesting a potentially novel treatment approach for endothelial barrier dysfunction and microvascular leakage-related diseases. Additionally, we found that irisin neutralizing antibody pretreatment increased the levels of total cells and proteins in the BALF, aggravated tissue damage, and decreased $\mathrm{PaO}_{2}$ after LPS administration, suggesting that endogenous irisin plays an important role in regulating endothelial barrier function.

The destruction of endothelial barrier integrity is pivotal in the pathogenesis of ARDS, sepsis, anaphylaxis, and other diseases (25). The mechanisms underlying this increased endothelial permeability have multiple suggested hypotheses. First, endotoxin can directly cause endothelial cell shrinkage and indirectly damage vascular endothelial cells by activating inflammatory cells, toxic oxygen free radicals, peroxides, proteolytic enzymes, and cytokines (1). Second, endotoxin and inflammatory factors cause MLC phosphorylation and joining with actin. Neutrophils induce the phosphorylation of $\beta$-catenin and 


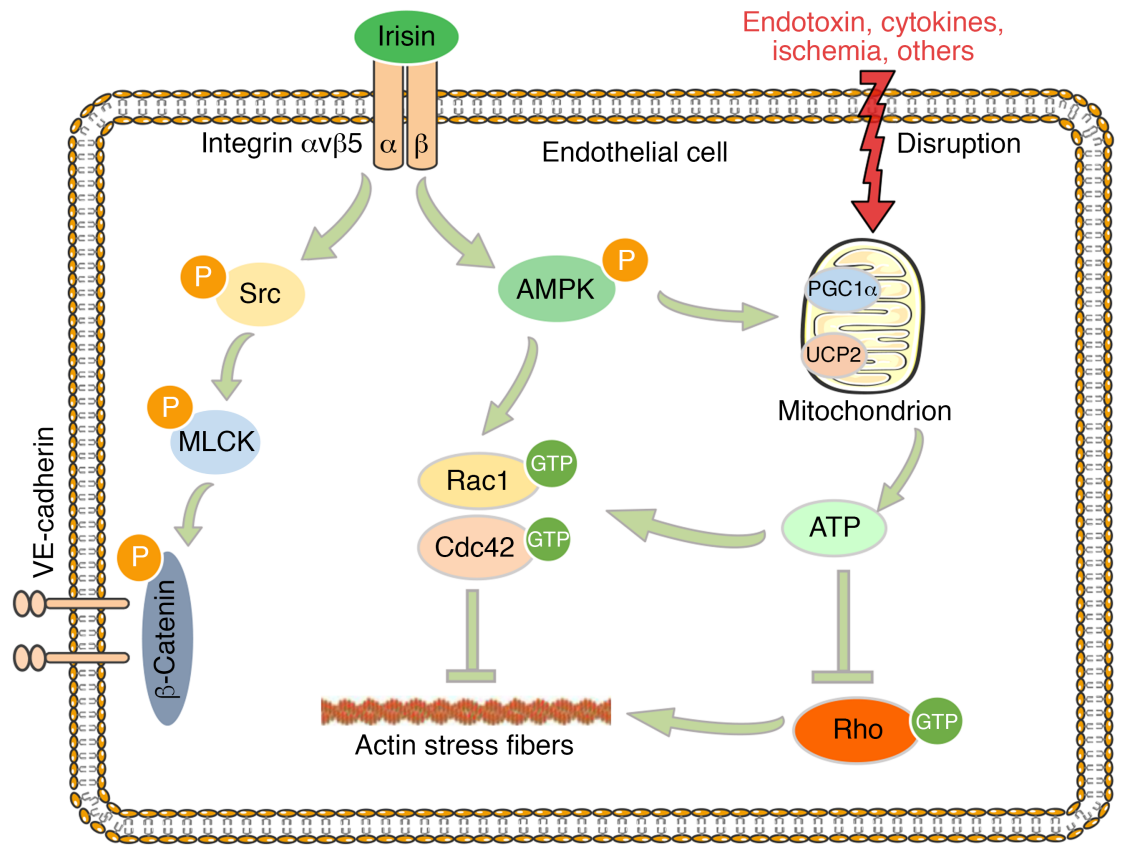

Figure 8. Exercise hormone irisin mitigates endothelial barrier dysfunction and microvascular leakage-related diseases.

the separation of VE-cadherin from the cytoskeleton, thereby weakening tight junctions between endothelial cells $(2,3)$. Moreover, the damage of endothelial progenitor cell (EPC) repair function in circulation results in the destruction of endothelial permeability (26).

Vascular integrins are major mediators of endothelial adhesion to extracellular matrix (13). SFK, especially Src, plays an important role in increasing permeability of endothelial cells under inflammatory conditions. Tyr416 (catalytic subunit localization) phosphorylation of Src enhances Src activity. A previous study has proved that Src directly alters the structure of the endothelial barrier by phosphorylation of MLCK, $\beta$-catenin, and focal adhesion (14). Src-deficient mice and inhibition of Src activity can reduce the degree of cerebral edema during stroke $(27,28)$. Interestingly, a recent study verified that integrin $\alpha \mathrm{V} \beta 5$ is the receptor of irisin in osteocytes and fat cells (12). However, whether irisin affects endothelial barrier function remains unclear. In this study, we found that irisin significantly decreased the activation of Src and inhibited the phosphorylation of MLCK, $\beta$-catenin. Irisin might enhanced endothelial barrier function via suppression of the P-Src (Y416)/P-MLCK (Y464)/P- $\beta$-catenin (Y142) pathway.

AMPK is a central metabolic sensor regulating energy metabolism and mitochondrial function (19). Meanwhile, it has been shown that AMPK activation can also protect vascular barrier function by strengthening endothelial intercellular junctions and cytoskeletal remodeling (18). Interestingly, several studies have reported that the regulatory role of irisin in energy metabolism is associated with activation of AMPK $(15,29)$. In the present study, irisin treatment significantly increased the activation of AMPK in endothelial cells. Meanwhile, administration of compound C or AMPK siRNA abolished the protective function of irisin in microvascular leakage in both in vitro and in vivo experiments. Our study indicates that irisin treatment might restore endothelial barrier function via activation of AMPK.

The Rho GTPase family, including Rho, Rac1, and Cdc42, regulates endothelial intercellular junctions and cytoskeletal remodeling (30). It has been shown that Rac1 maintains tight junctions of endothelial cells via the formation of cortical actin in its GTP-bound state (i.e., the active state; refs. 20, 31). Conversely, the GDP-bound state is associated with vascular leakage (32). AMPK activation can strengthen the aggregation of microtubules and myosin to protect vascular barrier function (18). In our study, we found that the activation of Rac1 and Cdc42 was increased after irisin administration. However, AMPK siRNA transfection reversed the increased activation of Rac1 and Cdc42 in irisin-treated HMVECs. Our results demonstrate that irisin may protect endothelial barrier function through activation of the AMPK-Rac1/Cdc42 pathway. 
Mitochondria regulate ATP synthesis, ROS production, apoptosis stimulation, and aging (33). Mitochondrial dysfunction is an important cause of endothelial barrier dysfunction (34). Maintaining sufficient quality and quantity of mitochondria in endothelial cells is an essential requirement for endothelial barrier integrity (6). Mitochondrial biogenesis is responsible for the generation of mitochondria, which is regulated by PGC-1 $\alpha$ and its downstream target TFAM $(35,36)$. Many studies have shown that PGC- $1 \alpha$ expression is regulated by AMPK activation $(37,38)$. Furthermore, ATP facilitates Rac activation and cortactin formation to exert endothelial barrier protection $(8,20)$. ATPB is a key enzyme in the process of ATP synthesis. Previous studies have shown that irisin protects mitochondrial function in instances of ischemia/reperfusion injury (23). Our study revealed that irisin treatment reversed the decreased expression of PGC-1 $\alpha$ and TFAM after LPS treatment, which was abolished by transfection with AMPK siRNA. MitoTracker staining showed that irisin treatment significantly increased mitochondria numbers. Additionally, irisin restored ATPB expression and ATP levels after LPS administration. Taken together, our results reveal that irisin may facilitate endothelial barrier function via facilitating AMPK/PGC-1 $\alpha$ signaling-dependent mitochondrial biogenesis and increasing ATP production.

Certain limitations should be considered regarding this study. On the one hand, the present study focused on the role of irisin in endothelial barrier function in lung injury. The effects of irisin on lung epithelial cells and any other pathogenic mechanisms require further exploration. On the other hand, although exogenous irisin protects against microvascular leakage by restoring lung endothelial barrier function, the therapeutic effects identified here are only based on basic experiments, and prospective clinical studies are needed.

In conclusion, irisin mitigates endothelial barrier dysfunction and microvascular leakage-related diseases via binding to integrin $\alpha \mathrm{V} \beta 5$ receptor, further inhibiting the P-Src (Y416)/P-MLCK (Y464)/P- $\beta$-catenin (Y142) pathway, activating the AMPK-Cdc42/Rac1 pathway and improving mitochondrial function in endothelial cells. Low serum irisin was associated with worse outcomes in ARDS patients, and exogenous irisin protected against microvascular leakage-related diseases. Irisin may, therefore, assist with the urgent medical need for preventing or minimizing microvascular leakage-related diseases.

\section{Methods}

Supplemental Methods are available online with this article.

Patients. This study included 60 adult ARDS patients (age $\geq 18$ year) admitted to the First Affiliated Hospital of Xi'an Jiaotong University. ARDS was defined as having $\mathrm{PaO}_{2} /$ fraction of inspired oxygen $\left(\mathrm{FiO}_{2}\right) \leq 300 \mathrm{mmHg}$, acute pulmonary infiltrates identified on chest x-ray or computed tomography and mechanical ventilation with a positive end-expiratory pressure (PEEP) of at least $5 \mathrm{cmH}_{2} \mathrm{O}(39)$. The severity of ARDS was assessed using the APACHE II score and the SOFA score. Patient survival was monitored for 28 days after admission. Sixty healthy volunteers who underwent routine physical examination were included as healthy controls.

Experimental animals. Experiments were performed on male WT C57BL/6J mice (aged 6-8 weeks, weighing 20-25 g) and male Sprague-Dawley rats (weighing 500-650 g, aged 22 months). All the animals were purchased from the Laboratory Animal Center of Xi'an Jiaotong University.

In this study, all the animals were anesthetized by inhaling 3\% isoflurane. Euthanasia was conducted by exsanguination and cervical dislocation under deep anesthesia with isoflurane in all animal experiments.

Statistics. All measurement data are expressed as the mean \pm SEM. The 2-tailed $t$ test was used to analyze the differences between 2 groups, and 1-way ANOVA was used to analyze the differences among 3 or more groups. Spearman's correlation coefficient $(\rho)$ was used to analyze associations between 2 parameters. Kaplan-Meier curves were used for survival analysis and log-rank testing for difference analysis. All analyses were conducted with data statistics software SPSS 18.0. $P<0.05$ represented a significant difference.

Study approval. The study was approved by the Ethics Committee of the First Affiliated Hospital of Xi'an Jiaotong University. All study participants provided informed consent in accordance with the Declaration of Helsinki. All animal experiments were performed in accordance with the guidelines of the China Council on Animal Care and Use and approved by the IACUC of the Ethics Committee of Xi'an Jiaotong University Health Science Center, China (approval no. 2017-564). All animal experiments conformed to the guidelines from Directive 2010/63/EU of the European Parliament on the protection of animals used for scientific purposes. 


\section{Author contributions}

JB participated in the research design, performed most experiments, statistical analysis and paper writing; $\mathrm{JZ}$ and YR, participated in the animal studies and Western blot analysis. ZD and YZ participated in the ELISA, statistical analysis and participated in the cell culture and immunofluorescence. CL, LZ, YW, and ZS collected the serum from patients and analyzed human data. ZW and YL assisted with the design of the study. RW designed and supervised the study and revised the manuscript. All authors have read and agreed with the submission of the manuscript.

\section{Acknowledgments}

This work was supported by the National Nature Science Foundation of China (81770491 to RW) and the Ministry of Education Innovation Team Development Program of China (IRT16R57 to YL). We thank Ying Hao at the Instrument Analysis Center of Xi'an Jiaotong University for her assistance with confocal analysis.

Address correspondence to: Rongqian Wu, National Local Joint Engineering Research Center for Precision Surgery \& Regenerative Medicine, The First Affiliated Hospital of Xi'an Jiaotong University, 76 West Yanta Road, P.O. Box 124, Xi'an, Shaanxi Province 710061, China. Phone: 86.29.82657541; Email: RongqianWu:rwu001@mail.xjtu.edu.cn.

1. Aird WC. The role of the endothelium in severe sepsis and multiple organ dysfunction syndrome. Blood. 2003;101(10):3765-3777.

2. Muller WA. Transendothelial migration: unifying principles from the endothelial perspective. Immunol Rev. 2016;273(1):61-75.

3. Harris ES, Nelson WJ. VE-cadherin: at the front, center, and sides of endothelial cell organization and function. Curr Opin Cell Biol. 2010;22(5):651-658.

4. Tzima E, et al. Activation of Rac1 by shear stress in endothelial cells mediates both cytoskeletal reorganization and effects on gene expression. EMBO J. 2002;21(24):6791-6800.

5. Huang RT, et al. Experimental Lung Injury Reduces Krüppel-like Factor 2 to Increase Endothelial Permeability via Regulation of RAPGEF3-Rac1 Signaling. Am J Respir Crit Care Med. 2017;195(5):639-651.

6. Pangare M, Makino A. Mitochondrial function in vascular endothelial cell in diabetes. J Smooth Muscle Res. 2012;48(1):1-26.

7. Xu W, et al. Alterations of cellular bioenergetics in pulmonary artery endothelial cells. Proc Natl Acad Sci USA. 2007;104(4):1342-1347.

8. Bongard RD, et al. Depleted energy charge and increased pulmonary endothelial permeability induced by mitochondrial complex I inhibition are mitigated by coenzyme Q1 in the isolated perfused rat lung. Free Radic Biol Med. 2013;65:1455-1463.

9. Froehlich G, Crake T, Meier P. Exercise training for refractory angina: improving the coronary collateral circulation. Cardiology. 2012;123(2):78-9; author reply 80.

10. Boström P, et al. A PGC1- $\alpha$-dependent myokine that drives brown-fat-like development of white fat and thermogenesis. Nature. 2012;481(7382):463-468.

11. Panati K, Suneetha Y, Narala VR. Irisin/FNDC5--An updated review. Eur Rev Med Pharmacol Sci. 2016;20(4):689-697.

12. Kim H, et al. Irisin Mediates Effects on Bone and Fat via $\alpha$ V Integrin Receptors. Cell. 2018;175(7):1756-1768.e17.

13. Zhao X, Guan JL. Focal adhesion kinase and its signaling pathways in cell migration and angiogenesis. Adv Drug Deliv Rev. 2011;63(8):610-615.

14. Hu G, Minshall RD. Regulation of transendothelial permeability by Src kinase. Microvasc Res. 2009;77(1):21-25

15. Tang H, Yu R, Liu S, Huwatibieke B, Li Z, Zhang W. Irisin Inhibits Hepatic Cholesterol Synthesis via AMPK-SREBP2 Signaling. EBioMedicine. 2016;6:139-148.

16. Liu J, Song N, Huang Y, Chen Y. Irisin inhibits pancreatic cancer cell growth via the AMPK-mTOR pathway. Sci Rep. 2018;8(1):15247.

17. Fisslthaler B, Fleming I. Activation and signaling by the AMP-activated protein kinase in endothelial cells. Circ Res. 2009;105(2):114-127.

18. Xing J, Wang Q, Coughlan K, Viollet B, Moriasi C, Zou MH. Inhibition of AMP-activated protein kinase accentuates lipopolysaccharide-induced lung endothelial barrier dysfunction and lung injury in vivo. Am J Pathol. 2013;182(3):1021-1030.

19. Herzig S, Shaw RJ. AMPK: guardian of metabolism and mitochondrial homeostasis. Nat Rev Mol Cell Biol. 2018;19(2):121-135.

20. Jacobson JR, Dudek SM, Singleton PA, Kolosova IA, Verin AD, Garcia JG. Endothelial cell barrier enhancement by ATP is mediated by the small GTPase Rac and cortactin. Am J Physiol Lung Cell Mol Physiol. 2006;291(2):L289-L295.

21. Zhang HJ, et al. Irisin is inversely associated with intrahepatic triglyceride contents in obese adults. J Hepatol. 2013;59(3):557-562.

22. Perakakis N, et al. Physiology and role of irisin in glucose homeostasis. Nat Rev Endocrinol. 2017;13(6):324-337.

23. Chen K, et al. Irisin protects mitochondria function during pulmonary ischemia/reperfusion injury. Sci Transl Med. 2017;9(418):eaao6298.

24. Shao L, Meng D, Yang F, Song H, Tang D. Irisin-mediated protective effect on LPS-induced acute lung injury via suppressing inflammation and apoptosis of alveolar epithelial cells. Biochem Biophys Res Commun. 2017;487(2):194-200.

25. Matthay MA, Zemans RL. The acute respiratory distress syndrome: pathogenesis and treatment. Annu Rev Pathol. 2011;6:147-163.

26. Zhao YD, et al. Bone marrow progenitor cells induce endothelial adherens junction integrity by sphingosine-1-phosphate-mediated Rac1 and Cdc42 signaling. Circ Res. 2009;105(7):696-704.

27. Paul R, et al. Src deficiency or blockade of Src activity in mice provides cerebral protection following stroke. Nat Med. $2001 ; 7(2): 222-227$. 
28. Weis S, et al. Src blockade stabilizes a Flk/cadherin complex, reducing edema and tissue injury following myocardial infarction. J Clin Invest. 2004;113(6):885-894.

29. Xin C, et al. Irisin improves fatty acid oxidation and glucose utilization in type 2 diabetes by regulating the AMPK signaling pathway. Int J Obes (Lond). 2016;40(3):443-451.

30. Cullere X, Shaw SK, Andersson L, Hirahashi J, Luscinskas FW, Mayadas TN. Regulation of vascular endothelial barrier function by Epac, a cAMP-activated exchange factor for Rap GTPase. Blood. 2005;105(5):1950-1955.

31. Su G, et al. Absence of integrin $\alpha v \beta 3$ enhances vascular leak in mice by inhibiting endothelial cortical actin formation. $A m J$ Respir Crit Care Med. 2012;185(1):58-66.

32. Vandenbroucke E, Mehta D, Minshall R, Malik AB. Regulation of endothelial junctional permeability. Ann N Y Acad Sci. 2008;1123:134-145

33. McBride HM, Neuspiel M, Wasiak S. Mitochondria: more than just a powerhouse. Curr Biol. 2006;16(14):R551-R560

34. Szewczyk A, et al. Mitochondrial mechanisms of endothelial dysfunction. Pharmacol Rep. 2015;67(4):704-710.

35. Handschin C, Spiegelman BM. Peroxisome proliferator-activated receptor gamma coactivator 1 coactivators, energy homeostasis, and metabolism. Endocr Rev. 2006;27(7):728-735.

36. Scarpulla RC. Transcriptional paradigms in mammalian mitochondrial biogenesis and function. Physiol Rev. 2008;88(2):611-638.

37. Liu J, et al. Coupling of mitochondrial function and skeletal muscle fiber type by a miR-499/Fnip1/AMPK circuit. EMBO Mol Med. 2016;8(10):1212-1228.

38. Viscomi C, et al. In vivo correction of COX deficiency by activation of the AMPK/PGC-1 $\alpha$ axis. Cell Metab. 2011;14(1):80-90.

39. Fan E, Brodie D, Slutsky AS. Acute Respiratory Distress Syndrome: Advances in Diagnosis and Treatment. JAMA. 2018;319(7):698-710. 